\title{
CORRIGENDUM
}

doi:10.1038/nature08771

\section{FGF signalling during embryo development regulates cilia length in diverse epithelia}

Judith M. Neugebauer, Jeffrey D. Amack, Annita G. Peterson,

Brent W. Bisgrove \& H. Joseph Yost

Nature 458, 651-654 (2009)

In Figure 4 of this Letter, panel $\mathbf{b}(f g f r 1 \mathrm{MO}$, sox17 in situ) was inadvertently duplicated in panel a (WT uninj., sox17 in situ) during figure revision and assembly. The corrected Figure panels are shown below. This does not alter the data indicating that sox 17 expression is not affected in $f_{g} f r 1 \mathrm{MO}$, or any of the conclusions of the work presented in the manuscript.
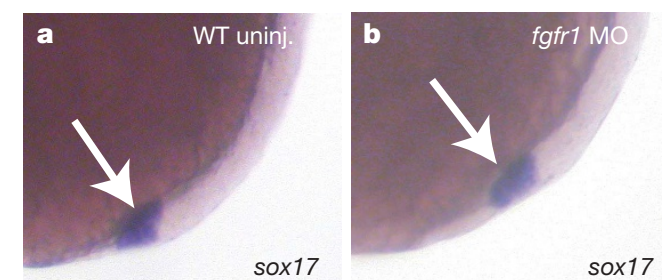